SELECTED PAPER AT NCSP'20

\title{
Workspace Enlargement of Cable-Based Haptic Device Using Linear Actuator
}

\author{
Bastien Poitrimol ${ }^{1}$ and Hiroshi Igarashi ${ }^{2}$ \\ Department of Electrical and Electronic Engineering, Tokyo Denki University \\ 5 Senju-Asahi-cho, Adachi-ku, Tokyo 120-8551, Japan \\ ${ }^{1}$ E-mail: b.poitrimol@crl.epi.dendai.ac.jp \\ ${ }^{2}$ E-mail: h.igarashi@mail.dendai.ac.jp
}

\begin{abstract}
Haptic displays have been attracting attention for several decades and a broad range of devices have been developed to allow interaction with virtual objects. Among these, cablebased architectures were researched in order to overcome the lack of flexibility and the intrusiveness inherent to rigid haptic displays. Cable robots allow the utilization of several end effectors, which can be proved to be particularly useful for providing haptic rendering on several fingers for the bimanual manipulation of virtual objects. Nonetheless, interference can appear when cables touch each other during manipulation. Also, some configurations may require a high number of cables to make full use of the frame of the device. Here, we propose a novel architecture of a haptic display based on SPIDAR. Our purpose is to enlarge the usable workspace of an existing device configuration while lowering the number of cables and without hindering the quality of the display. A hybrid planar haptic device architecture with $n+1$ cables that includes a linear module is introduced. Design considerations and the kinematics used are detailed. Then, simple tasks are performed to verify the usability of the device as a haptic display.
\end{abstract}

\section{Introduction}

Cable-driven parallel robots were primarily developed to carry out tasks that robots with rigid links could not handle [1]. Also, with the spread of virtual reality (VR) in our everyday lives, interest in letting people interact with these virtual worlds arose. VR can be described as an immersive experience that makes the user interact with artificially displayed objects. Coupling VR and haptic rendering followed naturally since the lack of force feedback was a hinderance in trying to provide a realistic experience.

The first interfaces were indeed, robots with rigid links such as the well-known stylus-based PHANToM [2]. However, cable-based interfaces such as SPIDAR [3], were created to make the most of the interesting features they provide. These interfaces have a low mass and a high potential reconfigurability, which helps modify the workspace, and also a low intrusiveness (the cables are less notable) for better immersion [4]. Cable devices have been used for many applications but have not changed markedly despite numerous evolutions. For instance, SPIDAR became a bimanual interface that can display forces at the tips of multiple fingers at the same time [5].

Regardless of their advantages, cable-based haptic devices share the same drawbacks. First, the system should be overconstrained: the number of cables is equal to the number of degrees of freedom +1 [6]. In the case of SPIDAR, the system struggles to allow both rotations and translations. Also, the cable tension has to remain positive and the workspace where forces can be displayed is always smaller than the span of the device.

The purpose of this research is to enlarge the reachable workspace of a planar haptic device with $n+1$ cables using a linear actuator to match the workspace of a square planar robot with $n+2$ cables. In the future, we plan to adapt the concept to a 3D system. Thus, we aim to reduce the size of the device and avoid potential interference between cables when using several effectors, such as in SPIDAR.

\section{Cable Robot and Workspace}

\subsection{Workspace: 2D and 3D configurations}

The reconfigurability and simple structure of cable-based interfaces are key factors when the workspace needs to be enlarged. Because of these factors, human-size virtual environments can be created easily and experienced with highquality haptic rendering [7]. Yet sometimes the size cannot be increased freely, for example when a small interface that can fit on a desk is required. While rigid interfaces such as PHANToM can be used on a desk as micromanipulation or teleoperation interfaces, cable robots are still lacking in this field. Also, the classic SPIDAR configuration with four cables per finger can use only half of the space inside the frame for haptic rendering. If it had eight cables, the system would gain in precision and all the sapce would be usable. However, this would prevent the user from reaching the end effector 


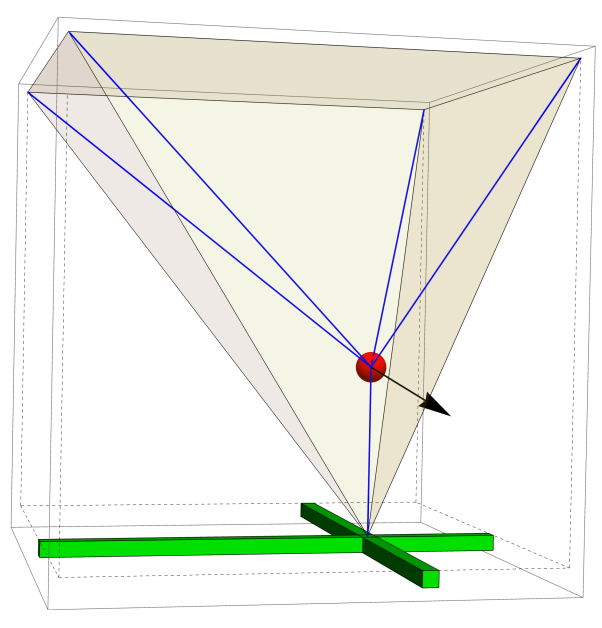

Figure 1: Representation of the 3D configuration: the orange pyramid corresponds to the workspace

easily and cause interference between cables.

To optimize the use of a workspace, we propose a design that includes a linear module that moves the attainable workspace dynamically during manipulation. The device functions exactly like SPIDAR when displaying force feedback. When the user is not touching a virtual object, the actuator follows the end effector so that one of the cables is always perpendicular to the ground. Figure 1 is a representation of the proposed configuration in three dimensions and its impact on the workspace. In this case, only the z-coordinate of the fifth cable varies. Such a configuration adds only one cable per finger and is perfectly compatible with a potential upgrade that can use multiple fingers and perform pick and place tasks.

Our approach is to apply the above-mentioned features to a robot with $n+1$ cables [6], which will serve as a proof of concept. A two-dimensions design was chosen to avoid more complex design considerations and because it can be upgraded to a 3D system with relative ease. The expected results of our approach is the enlargement of the workspace such that the entire span of the square frame can be reached when using only three cables.

\subsection{Proposed system}

The system shown in Fig. 2 was designed so that it fits on a desk and the flowchart of the data is presented in Fig.3. Considering the constraints outlined earlier, choosing hightorque direct-drive motors helps avoid a backlash. Moreover, the ability to display a wide range of forces on the handle to reproduce the sensation of touching a hard object, such as a wall, helps to evaluate the quality of the haptic rendering.

The interface includes four motors: three direct-drive brushless DC motors (Maxon EC 90 mm diameter 260W con-

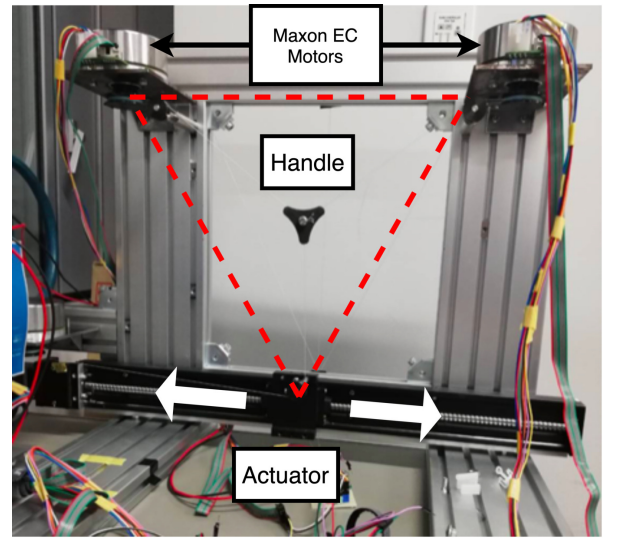

Figure 2: Planar cable-based haptic interface designed for this work

trolled with the corresponding 50/5 ESCON modules) used to control the cables and one AC servo motor, which controls the linear actuator (Misumi LMX-30, driven by a Mitsubishi HF-KP13 AC servo motor and MR-J3-10A).

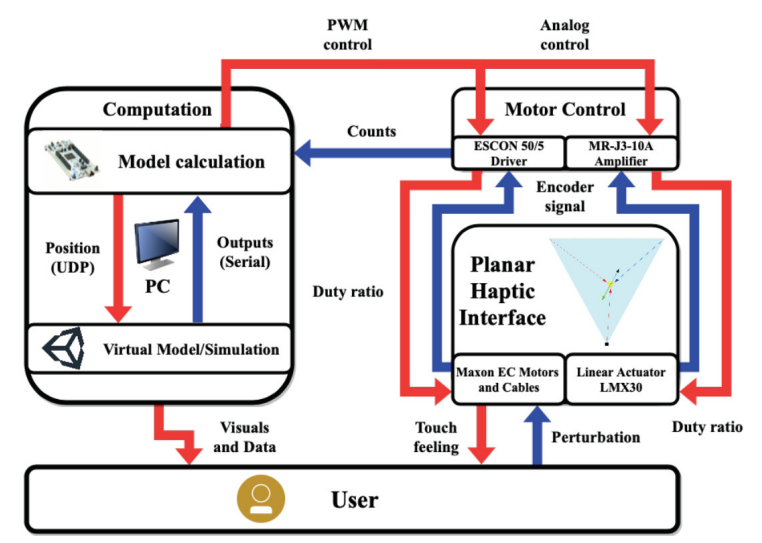

Figure 3: Flowchart of data used to emulate haptic rendering

Its dimensions are $50 \mathrm{~cm}$ in length, $30 \mathrm{~cm}$ in width and $40 \mathrm{~cm}$ in height. The manipulation workspace is about $30 *$ $30 \mathrm{~cm}^{2}$ and perpendicular to the ground to gain some space and to match the screen display. We plan to improve the virtual space display by using a VR headset.

\section{Model}

\subsection{Kinematic model}

The kinematic model in figure 4 has commonly been used in other studies and is largely inspired by the model introduced by Williams and Gallina [6]. The fixed length $A$ indi- 
cates the height and width of the frame. Each of the three cables passes through anchor points denoted by $\left\{M_{i} x M_{i} y\right\}^{T}$ with a variable length $L_{i}$ and angles $\theta_{i}(i=1,2,3)$. The components of their position vectors are expressed as $\left(M_{1}\right.$, $\left.X_{0}, Y_{0}\right)$ and the end effector $(x, y)$ is considered as a point. The coordinate system attached to the end effector is purely indicative as it is only used to simplify the formulas when matching the device to the simulation in Unity. $X_{L}$ corresponds to the position of the linear actuator. When initializing the system, the end effector is placed at the centroid of the triangle. This can be carried out precisely without requiring an advanced calibration method, in contrast to $3 \mathrm{D}$ systems [8].

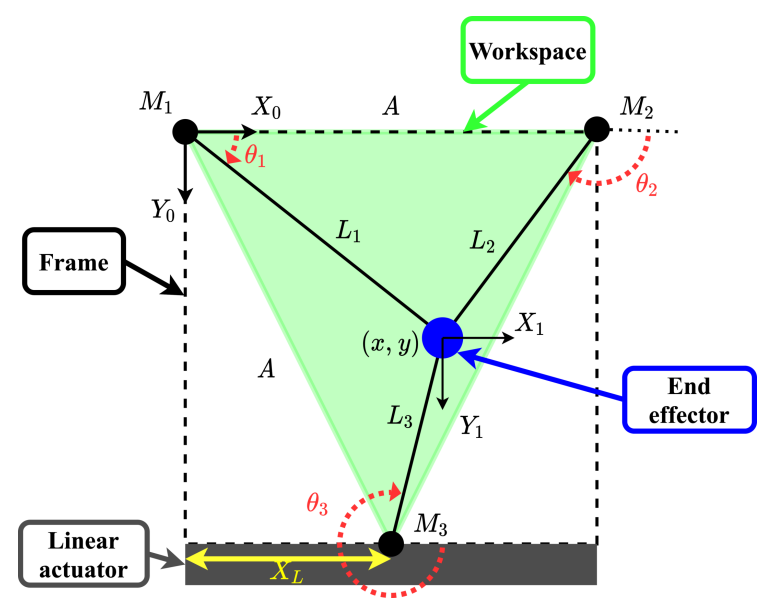

Figure 4: Kinematic model for planar configuration with $n+1$ cables

The position of the handle is obtained from each wire's length $L_{i}$ measured using the motor encoder. By writing equation 1 for each cable, a system of three equations (five in a 3D-system) with two unknowns is obtained.

$$
L_{i}=\sqrt{\left(x-M_{i} x\right)^{2}+\left(y-M_{i} y\right)^{2}}
$$

Then the $(x, y)$ coordinates of the end effector can be written knowing the lengths of two of the three cables as in equation (2). In our case, the data from the two first motors $\left(M_{1}\right.$ and $M_{2}$ ) are used owing to the fact that the $x$-coordinate of the linear actuator is matched with $M_{3}$.

$$
x=\frac{L_{1}^{2}-L_{2}^{2}+A^{2}}{2 A}, y=\sqrt{L_{2}-x^{2}}
$$

The cable angles are calculated and used in the Jacobian matrix to distribute the feedback between the cables. Note that only two out of the three cables are necessary for haptic feedback: the other one can be kept at a predefined minimal tension. The validity of the method was shown by Williams and Gallina [6] and it can also be applied in three dimensions to simplify and accelerate the computation [4].
With the 3D configuration, writing the system of equations 1 shows that the data from the fifth cable are necessary to determine the z-coordinate. This leads to the need to clearly separate the free movement and haptic feedback phases during operation. Indeed, even if the $z$-coordinate does not change the length of the cable is changed by the linear actuator. Thus, the control strategy presented and verified in the next section for two dimensions should be adjusted.

\subsection{Control strategy}

Figure 5 shows the control strategy we chose in a simplified manner, which can be described as follows. In the first step, the system is initialized with given coordinates and minimal tension in the cables. Then, the position of the end effector is inferred from the encoders' data. Lastly, the data are computed to follow a reaction-torque-based algorithm $(u$ is the current output of the controller and $u_{R T O B}$ the reaction torque). The kinematics presented earlier are used to feedback the controller and switch the linear actuator on or off. The switch represents the change between the free-movement and haptic rendering modes (see Fig.5). Finally, the visual feedback is provided to the user via a Unity simulation displayed on the screen. The virtual model corresponds to the behavior of the virtual object, which is a mass, damper and stiffness model. By changing the coefficients, the surface of the object can be modified to be make it softer or stiffer.

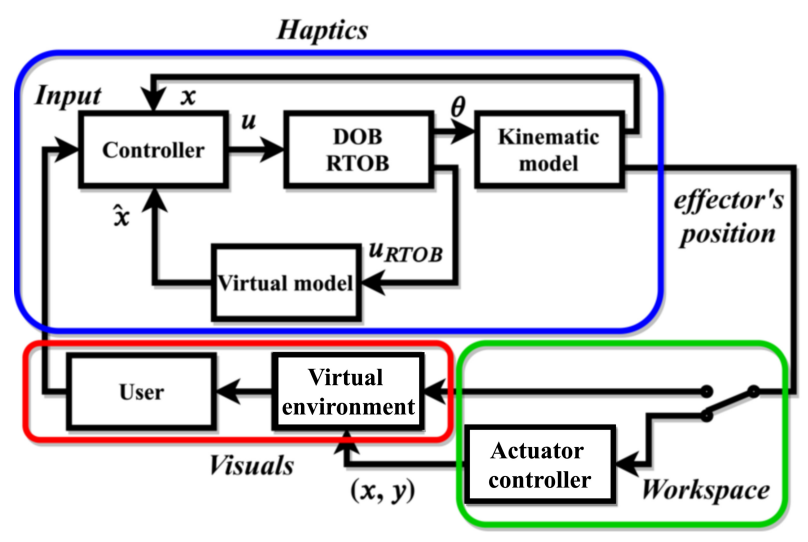

Figure 5: Simplified block diagram of the control strategy

The model can be improved by adding a second virtual model: thus, both the movement and texture of the object can be rendered. The full control algorithm is still being implemented. Thus far, the effect of the linear module on free movement has been investigated and the ability of the proposed control method to provide haptic feedback has been tested on a 1D task. Preliminary results are presented in the next section. 


\section{Results}

The ability of the system to provide the sense of touch has been verified by touching a virtual wall in a linear task. The results for this task are not presented since the focus is the workspace. Moreover, it is difficult to quantify the quality of the displayed forces but the contacts were convincing and had no vibrations. Moreover, the workspace of the device has been successfully enlarged using PID control on the actuator. Figure 6 shows the spaces where the user is not opposing all the cables tensions. It can be observed that most of the space can be reached. Furthermore, the end effector tends to stay in place even if the user lets it go, whereas it returns to the center of the space without the actuator.

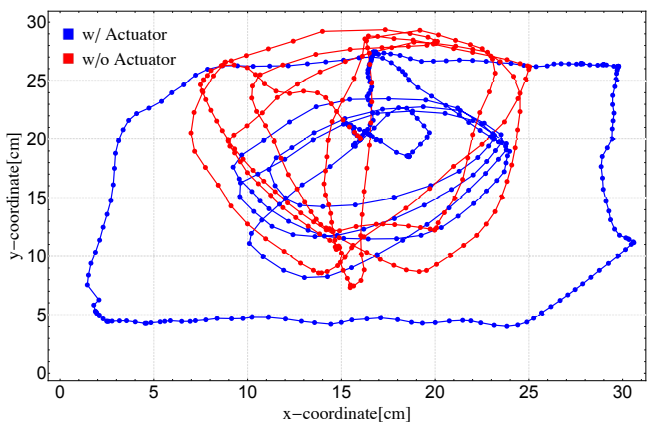

Figure 6: Workspace during free movement operation

Then, the effect of the actuator on the accuracy of manipulation during free movement was tested by following a trajectory, using Unity for the simulation (see figure 7). Neither an improvement nor a lower accuracy was observed, which at first glance suggests that the effect is minimal. However, on both the left and right sides of the graph, the red trajectory is wavy. This can be explained by the fact that the user has to go out of the workspace to reach the objective and thus oppose all the tensions maintaining the cables. This proves that the addition of the actuator has a positive effect during free movement because it keeps the effector farther from the limit of the workspace.

\section{Conclusions}

A novel architecture that uses a linear actuator for cablebased haptic interfaces has been introduced. The effect of the actuator on the free movement of an end effector was investigated. The device showed promising results in enlarging the usable workspace. Also, the proposed configuration reduced the noise induced by the minimal tension necessary to prevent the cables from slackening. A simple control scheme was tested on a $1 \mathrm{D}$ task and will be extended to $2 \mathrm{D}$ applications.

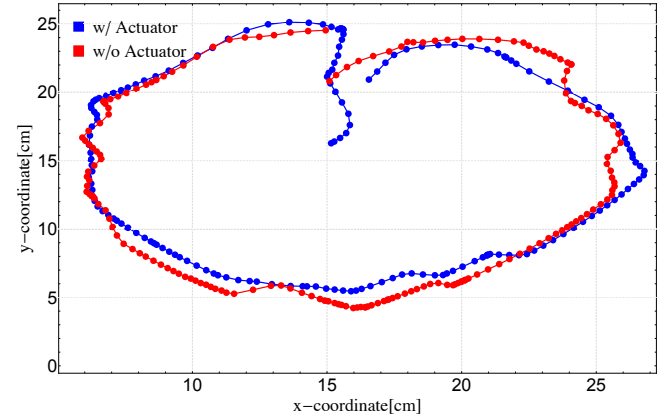

Figure 7: Circular trajectory followed with no feedback

Future works will include improvements to the control scheme as well as combining the device with a VR display to reinforce the immersion process. The final step will be to apply the concept to a SPIDAR-like 3D configuration.

\section{References}

[1] J. Albus, R. Bostelman and N. Dagalakis: The NIST SPIDER, A robot crane, Journal of Research of the National Institute of Standards and Technology, 1992.

[2] T. H. Massie and J. K. Salisbury: The PHANTOM haptic interface: A device for probing virtual objects, Proc. of the ASME Winter Annual Meeting, Symposium on Haptic Interfaces for Virtual Environment and Teleoperator Systems, Chicago, 1994.

[3] Y. Hirata and M. Sato: 3-dimensional interface device for virtual work space, Proc. of the IEEE/RSJ International Conference on IROS, 2, pp. 889-896, 1992.

[4] J. Saint-Aubert, S. Regnier and S. Haliyo: Cable driven haptic interface for co-localized desktop VR, IEEE Haptics Symposium (Haptics), San Francisco, CA, pp. 351-356, 2018.

[5] M. Sato: SPIDAR and virtual reality, Proc. of the 5th Biannual World Automation Congress, Orlando, FL, pp. 17-23, 2002.

[6] R. L. Williams II and P. Gallina: Translational planar cable-directdriven robots, Journal of Intelligent and Robotic Systems, Vol.37, pp. 69-96, 2003.

[7] A. Fortin-Cote, P. Cardou and C. Gosselin: An admittance control scheme for haptic interfaces based on cable-driven parallel mechanisms, IEEE International Conference on Robotics and Automation (ICRA), Hong Kong, pp. 819-825, 2014.

[8] P. Boudoin, H. Maaref, S. Otmane and M. Mallem: SPIDAR calibration based on neural networks versus optical tracking, 6th International Workshop on Artificial Neural Networks and Intelligent Information Processing (ANNIIP 2010), Funchal, France, pp. 87-98, 2010. 